\title{
Rastreio da Diabetes em Doentes com Doença \\ Macrovascular Coronária: As Novas Guidelines \\ Europeias são um Retrocesso?
}

\author{
Diabetes Screening in Patients with Macrovascular \\ Coronary Disease: Are the New European Guidelines a \\ Step Backwards?
}

\author{
Andreia RIBEIRO ${ }^{1}$, Sérgio Bravo BAPTISTA $\triangle^{2}$, Mariana FAUSTINO², Paulo ALVES ${ }^{2}$, Pedro Farto e ABREU², \\ Victor Machado $\mathrm{GIL}^{2}$, Carlos MORAIS ${ }^{2}$ \\ Acta Med Port 2017 Jun;30(6):434-442 - https://doi.org/10.20344/amp.7990
}

\begin{abstract}
RESUMO
Introdução: As novas recomendações europeias de diabetes mellitus tipo 2 e doença cardiovascular sugerem que o risco de diabetes mellitus tipo 2 deve ser avaliado através do score de risco FINnish Diabetes RIsk SCore e que o rastreio de diabetes mellitus tipo 2 na população com doença arterial coronária deve ser efetuado apenas com a glicemia plasmática em jejum e a $\mathrm{HbA1}$, remetendo a prova de tolerância oral à glicose para os casos 'inconclusivos'. Pretendemos avaliar os resultados desta estratégia, que difere da previamente defendida nas guidelines.
\end{abstract}

Material e Métodos: A glicemia plasmática em jejum, HbA1c e a prova de tolerância oral à glicose (75 g, 2 horas) foram avaliadas prospectivamente num grupo de doentes consecutivos submetidos a intervenção coronária percutânea, sendo usada a classificação da ADA para pré-diabetes mellitus tipo 2 e diabetes mellitus tipo 2. O risco de diabetes foi avaliado de acordo com o FINnish Diabetes Rlsk SCore.

Resultados: Foram incluídos 135 doentes (idade média 62,3 +/- 13,1 anos; 99 homens). Usando a prova de tolerância oral à glicose e a HbA1c, foram diagnosticados 18 (13,3\%) novos casos de diabetes mellitus tipo 2 e $77(57,0 \%)$ casos de pré-diabetes mellitus tipo 2. A glicemia plasmática em jejum + HbA1c identificou 12/18 doentes com diabetes mellitus tipo 2 (Sens 66,7\%; valor preditivo negativo 95,1\%; Kappa 0,78; $p<0,0001$ ) e 83 do total (pré-diabetes mellitus tipo 2/ diabetes mellitus tipo 2) de 95 doentes com distúrbios da glucose (Sens 87,4\%; valor preditivo negativo 76,9\%). Realizar adicionalmente prova de tolerância oral à glicose nos 29 doentes com um FINnish Diabetes RIsk SCore elevado permitiu diagnosticar 15/18 doentes com diabetes mellitus (Sens 83,3\%; valor preditivo negativo 97,5\%; Kappa 0,85; $p<0,0001$ ) e 86/95 dos doentes com distúrbios da glucose (Sens 90,5\%; valor preditivo negativo 81,6\%). Discussão: Apesar da melhoria diagnóstica, um em cada seis doentes com diabetes mellitus tipo 2 não seria diagnosticado por esta estratégia.

Conclusão: A utilização do FINnish Diabetes RIsk SCore como forma de selecionar os doentes candidatos a rastreio com prova de tolerância oral à glicose melhora a capacidade diagnóstica, quando comparada com a simples avaliação da glicemia plasmática em jejum e da HbA1c. No entanto, um em cada seis doentes com diabetes mellitus tipo 2 não é identificado com esta metodologia.

Palavras-chave: Diabetes Mellitus Tipo 2; Doença da Artéria Coronária; Hemoglobina A Glicosilada; Rastreio; Teste de Tolerância a Glucose

\section{ABSTRACT}

Background: The new European guidelines on diabetes mellitus and cardiovascular diseases propose that the FINnish Diabetes RIsk SCore should be used to evaluate the risk of diabetes mellitus and that diabetes mellitus screening in coronary artery disease patients should be based on fasting glucose and HbA1c. The 2 hour oral glucose tolerance test, recommended for all pts in the previous guidelines, is now only recommended for 'inconclusive' cases. We aimed to evaluate this new strategy.

Material and Methods: Fasting glucose, HbA1c and glucose tolerance test $(75 \mathrm{~g}, 2 \mathrm{~h})$ were prospectively evaluated in a consecutive group of pts with coronary artery disease. ADA criteria (both glucose tolerance test and $\mathrm{HbA} 1 \mathrm{c}$ ) were used to define diabetes mellitus and pre-diabetes mellitus. Diabetes mellitus risk was evaluated according to the FINnish Diabetes RIsk SCore.

Results: A total of 135 patients were included (mean age $62.3+/-13.1$ years, 99 males). Glucose tolerance test and $\mathrm{HbA} 1 \mathrm{c}$ together diagnosed $18(13.3 \%)$ new cases of diabetes mellitus and $77(57.0 \%)$ patients with pre-diabetes mellitus. Fasting glucose $+\mathrm{HbA} 1 \mathrm{c}$ (guidelines strategy) identified 12/18 patients with diabetes mellitus (Sens 66.7\%; negative predictive value 95.1\%; Kappa 0.78; $p<$ 0.0001 ) and $83 / 95$ patients with glucose anomalies (pre- diabetes mellitus + diabetes mellitus) (Sens $87.4 \%$; negative predictive value $76.9 \%)$. Performing glucose tolerance test in the 29 patients with an elevated FINnish Diabetes RIsk SCore would allow identifying $15 / 18$ patients with diabetes mellitus (Sens $83.3 \%$; negative predictive value $97.5 \%$; Kappa $0.85 ; p<0.0001$ ) and $86 / 95$ patients with glucose anomalies (Sens $90.5 \%$; negative predictive value $81.6 \%$ ).

Discussion: Although this strategy improved the screening accuracy, one in each six patients with diabetes mellitus would still remain undiagnosed, as compared to measuring $\mathrm{HbA} 1 \mathrm{c}$ and performing an glucose tolerance test in all patients.

Conclusion: Using the FINnish Diabetes RIsk SCore to select candidates to additional glucose tolerance test improves the accuracy for identifying diabetic patients, as compared with fasting glucose + HbA1c alone. However, 1/6 patients diabetes mellitus is still left undiagnosed with this strategy proposed by the current guidelines.

Keywords: Coronary Artery Disease; Diabetes Mellitus, Type 2; Glucose Tolerance Test; Hemoglobin A, Glycosylated; Mass Screening

1. Faculdade de Medicina. Universidade de Lisboa. Lisboa. Portugal.

2. Serviço de Cardiologia. Hospital Fernando Fonseca. Amadora. Portugal.

$\triangle$ Autor correspondente: Sérgio Baptista. sergio.b.baptista@gmail.com

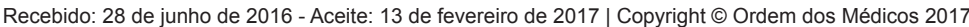




\section{INTRODUÇÃO}

Apesar do número de diabéticos estar a aumentar, estima-se que cerca de $30 \%$ da população com diabetes mellitus tipo 2 (DM) não se encontra diagnosticada e que $25 \%$ destes casos já têm retinopatia ou microalbuminúria estabelecida quando são diagnosticados. ${ }^{1,2}$ Embora a DM tenha sido definida em função do nível de hiperglicemia para o qual existe aumento do risco de doença microvascular (como retinopatia, nefropatia ou neuropatia), a presença de doença macrovascular, em particular doença arterial coronária (DAC), é muito relevante nos doentes diabéticos, porque condiciona de forma determinante o seu prognóstico. ${ }^{3}$

Os distúrbios do metabolismo da glicemia, por outro lado, são muito prevalentes, mas passam frequentemente despercebidos nestes doentes. ${ }^{4,5}$ Também este é um aspeto particularmente relevante, uma vez que as alterações no metabolismo da glicose se associam a um pior prognóstico nos doentes com DAC, quando comparados com doentes normoglicemicos. ${ }^{6-9}$

Compreendendo este impacto mútuo que a DAC e a diabetes têm, a Sociedade Europeia de Cardiologia (European Society of Cardiology - ESC) e a Associação Europeia para o Estudo da Diabetes (European Association for the Study of Diabetes - EASD) publicaram em 2007 a primeira edição das recomendações para o diagnóstico e da diabetes, pré-diabetes na presença de doença cardiovascular. ${ }^{10}$ Nestas guidelines, foi pela primeira vez assumida a necessidade de rastrear os distúrbios do metabolismo da glicemia (incluindo diabetes, mas também alteração da glicemia em jejum e diminuição da tolerância oral à glicose) em todos os doentes com DAC, recomendando-se a avaliação da glicemia em jejum complementada por uma prova de tolerância oral à glicose (PTOG) em todos os doentes coronários sem diabetes prévia.

Desde então, nova evidência surgiu e a $\mathrm{HbA} 1 \mathrm{c}$ passou a ser considerada como critério diagnóstico da diabetes. ${ }^{11-14}$ No sentido de incorporar a nova evidência, as guidelines europeias foram revistas em $2013,{ }^{3}$ passando a recomendar, nos doentes com doença cardiovascular estabelecida, apenas a avaliação da glicose plasmática em jejum (GPJ) e da HbA1c. De acordo com este novo documento, a PTOG é portanto apenas indicada para os casos 'inconclusivos', não sendo claro como deverão estes casos ser identificados. Em doentes sem DAC estabelecida, as novas guidelines recomendam ainda que o risco de diabetes seja calculado utilizando o score de risco FINnish Diabetes RIsk SCore (FINDRISC), que, em boa verdade, não foi validado na população de doentes com DAC. ${ }^{15}$ Esta estratégia difere da previamente recomendada, não sendo claro que percentagem de doentes deixará de ser diagnosticada se a PTOG não for efetuada de forma sistemática na população de doentes com DAC.

Foram objetivos deste estudo: 1) Avaliar a capacidade diagnóstica da estratégia proposta pelas guidelines europeias na identificação de novos casos de DM numa população de doentes com DAC estabelecida; 2) Avaliar o valor do score FINDRISC na seleção dos doentes com DAC can- didatos a PTOG.

\section{MATERIAL E MÉTODOS \\ Desenho do estudo e população de doentes}

Estudo observacional prospetivo, incluindo doentes consecutivos submetidos a angioplastia coronária e seguidos na consulta de Cardiologia de um único operador entre janeiro de 2013 e julho de 2014. Foram registados as principais características epidemiológicas (idade, sexo), físicas (índice de massa corporal e perímetro abdominal), clínicas (incluindo os fatores de risco cardiovasculares: hipertensão arterial, dislipidemia e tabagismo) e relacionadas com a doença coronária (número de vasos com doença, antecedentes de enfarte ou de revascularização). O risco de desenvolver diabetes foi avaliado pelo score de risco de FINDRISC para cada doente e a presença de distúrbios da glicemia foi avaliada pela GPJ, PTOG e HbA1c em todos os doentes.

\section{Score de risco de FINDRISC}

O score de risco FINDRISC $^{15}$ foi calculado em cada doente, incluindo as seguintes variáveis: idade, perímetro abdominal, IMC, hábitos de exercício físico, hábitos alimentares, história de HTA, distúrbios da glicemia previamente conhecidos e história familiar de DM (Fig. 1). O risco total foi calculado somando o valor dos parâmetros individuais, sendo expresso em termos de probabilidade de vir a desenvolver DM nos próximos 10 anos:

- Baixo risco: Score $<7$ (1/100 doentes irá desenvolver DM);

- Ligeiramente elevado: Score entre 7 e 11 (1/25 irá desenvolver DM);

- Moderado: Score entre 12 e 14 (1/6 irá desenvolver DM);

- Alto: Score entre 15 e 20 (1/3 irá desenvolver DM);

- Muito alto: Score > 20 (estima-se que 1 em 2 irá desenvolver DM).

\section{Avaliação laboratorial e critérios de diagnóstico da dia- betes}

$\mathrm{Na}$ primeira consulta após a angioplastia coronária (aproximadamente dois meses depois da alta hospitalar), foram requisitadas análises a todos os doentes sem diabetes conhecida. Estas análises foram realizadas entre o quarto e quinto mês após a alta hospitalar, e incluíram a GPJ, a PTOG e a HbA1c. A PTOG foi efetuada de acordo com as recomendações da OMS (GPJ, seguida de carga oral de 75 de glucose e de nova avaliação da glicemia aos 120 minutos após a carga). A HbA1c foi avaliada por cromatografia líquida de alta resolução na primeira colheita de sangue da PTOG. Os distúrbios da glicemia foram diagnosticados de acordo com as recomendações da American Diabetes Association (Tabela 1). ${ }^{16}$ Tal como descrito nessas recomendações, os doentes com anomalia da glicemia em jejum (AGJ) e com anomalia da tolerância à glicose (ATG) foram globalmente designados como pré-diabéticos. 


\section{FINish Diabetes Risk Score (FINDRISK) para avaliação do risco a} 10 anos da diabetes tipo 2 em adultos. (Disponível em: www.diabetes.fi/english)

\section{Formulário de avaliação de risco da diabetes tipo 2}

\section{Faça um círculo na alternativa correta e some os seus pontos.}

1. Idade

0 p. Até 45 anos

2 p. $45-54$ anos

3 p. $55-64$ anos

4 p. Mais de 64 anos

2. Índice de massa corporal

0 p. Inferior a $25 \mathrm{~kg} / \mathrm{m}^{2}$

1 p. $25-30 \mathrm{~kg} / \mathrm{m}^{2}$

3 p. Superior a $30 \mathrm{~kg} / \mathrm{m}^{2}$

3. Perímetro da cintura medida abaixo das costelas (geralmente ao nível do umbigo)

\section{HOMENS}

MULHERES

0 p. Menos de $94 \mathrm{~cm}$

3 p. $94-102 \mathrm{~cm}$

0 p. Menos de $80 \mathrm{~cm}$

4 p. Mais de $102 \mathrm{~cm}$

3 p. $80-88 \mathrm{~cm}$

4 p. Mais de $88 \mathrm{~cm}$
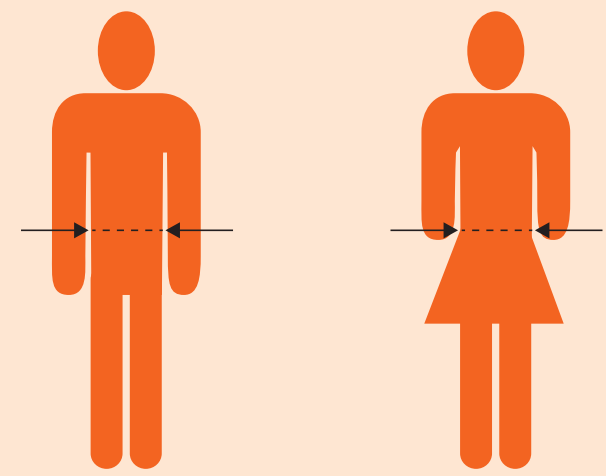

4. Costuma praticar actividade física pelo menos 30 min diários no trabalho e / ou durante o tempo de lazer

(incluindo a actividade diária normal)?

0 p. Sim

2 p. Não

5. Quantas vezes come vegetais, fruta ou frutos vermelhos?

0 p. Todos os dias

1 p. Não todos os dias
6. Já tomou regularmente medicamentos anti-depressivos?

0 p. Não

2 p. $\operatorname{Sim}$

7. Alguma vez lhe foi detectada a presença de níveis elevados de glicose no sangue (i.e. num exame médico, durante uma doença, durante a gravidez)?

0 p. Não

5 p. Sim

8. A algum dos seus familiares directos ou outros parentes foi diagnosticada diabetes (tipo 1 ou tipo 2)?

0 p. Não

3 p. Sim: avós, tia, tio, ou primo direito (mas não os próprios pais, irmão, irmã ou filho)

5 p. Sim: pais, irmão, irmã ou filho

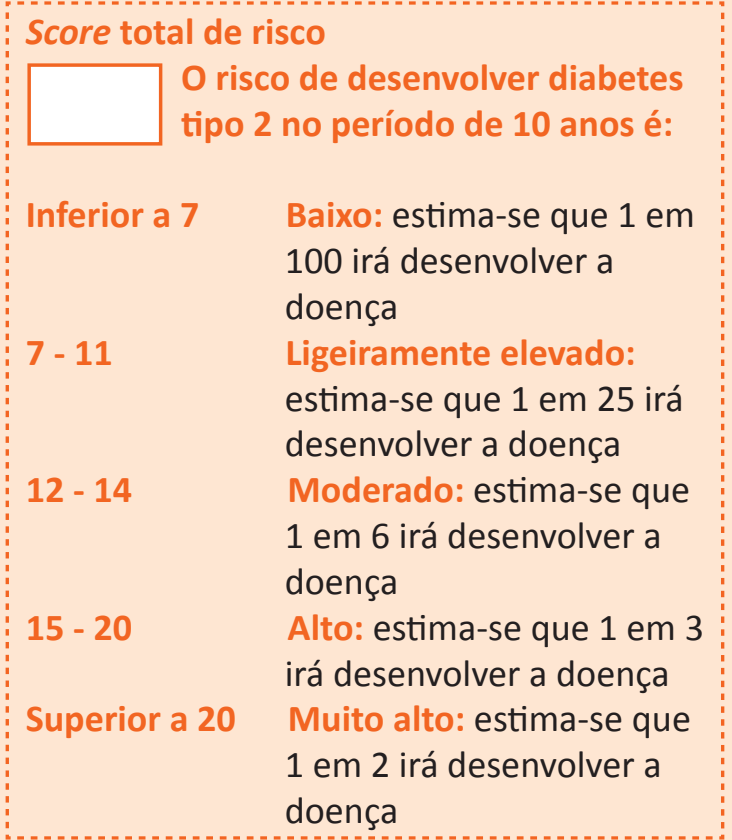

Teste concebido pelo Professor Jaakko Tuomilehto, Departamento de Saúde Pública, Universidade de Helsínquia e pela Dra Jaana Lindström, MFS, National Public Health Institute.

Figura 1 - Score FINish Diabetes Score (FINDRISC) ${ }^{3}$ 
Tabela 1 - Critérios de diagnóstico da diabetes (American Diabetes Association, 2016) ${ }^{16}$

\begin{tabular}{llccc}
\hline & & $\begin{array}{c}\text { Glicemia jejum } \\
(\mathrm{mg} / \mathrm{dL})\end{array}$ & $\begin{array}{c}\text { Glicemia 120 min } \\
(\mathrm{mg} / \mathrm{dL})\end{array}$ & HbA1c \\
\hline Normal & & $<100$ & $<140$ & $<5,7$ \\
\multirow{2}{*}{ Pré-diabetes } & Anomalia da glicemia em jejum (AGJ) & $100-126$ & $<140$ & $5,7-6,4$ \\
& Anomalia da tolerância à glicose (ATG) & $<126$ & $140-200$ & $>200$ \\
Diabetes & & $>126$ & $>6,5$ \\
\hline
\end{tabular}

\section{Análise estatística}

As variáveis contínuas com distribuição normal foram expressas como média e desvio padrão. As variáveis contínuas com distribuição não normal foram expressas como mediana e intervalo inter-quartil. A normalidade foi avaliada através dos testes de Kolmogorov-Smirnov e da avaliação visual dos gráficos $Q-Q$. As variáveis discretas foram expressas como percentagens. As comparações entre as variáveis dos diferentes grupos avaliados (classificação dos distúrbios da glicemia e grupos de risco pelo score FINDRISK) foram efetuadas por teste de $\chi 2$, para as variáveis categóricas o teste $t$-Student ou one-way ANOVA, para as variáveis contínuas com distribuição normal ou teste Kruskal-Wallis para as variáveis contínuas com distribuição não normal. A concordância entre as várias técnicas para avaliação dos distúrbios da glicemia foi avaliada através do cálculo do valor de Kappa. Para todas as comparações, um valor de $p<0,05$ foi considerado como estatisticamente significativo. A análise estatística foi efetuada utilizando o programa IBM ${ }^{\circledR}$ SPSS ${ }^{\circledR}$ Statistics version 19.0 (SPSS Inc., Chicago, IL).

\section{RESULTADOS}

\section{Características da população incluída}

De uma população inicial de 185 doentes com DAC estabelecida, 50 (27,0\%) foram excluídos do estudo por apresentarem diagnóstico prévio de DM (Fig. 2). As principais características da população, incluindo os fatores de risco cardiovascular, os principais antecedentes cardiovasculares, o perfil lipídico e as análises de diagnóstico da diabetes, são apresentadas na Tabela 2.

\section{Resultados em função da presença de distúrbios de glicemia}

A avaliação em conjunto da PTOG e HbA1c resultou no diagnóstico de $77(57 \%)$ casos de pré-diabetes e 18 (13\%) casos de DM. Apenas 40 (30\%) doentes não apresentavam distúrbios da glicemia (Fig. 2). Para além dos 50 doentes excluídos desta análise por já terem diabetes conhecida, foram identificados mais 18 doentes com diabetes não conhecida, o que significa que a prevalência total de diabetes foi de $36,8 \%$ (68/185). As características da população em função da presença de distúrbios da glicemia são apresentadas na Tabela 2. Os doentes com novo diagnóstico de DM tinham maior índice de massa corporal e uma tendência para maior perímetro abdominal, mas não se documentaram outras diferenças significativas, nomeadamente na idade e nos fatores de risco cardiovascular. Em termos analíticos, os doentes com DM e pré-diabetes tinham níveis mais elevados de triglicéridos, não se documentando diferenças nos restantes parâmetros do perfil lipídico. Os valores de glicemia, glicémia aos 120 minutos e HbA1c aumentaram de forma significativa entre os doentes com perfil glicémico normal, pré-diabetes e DM.

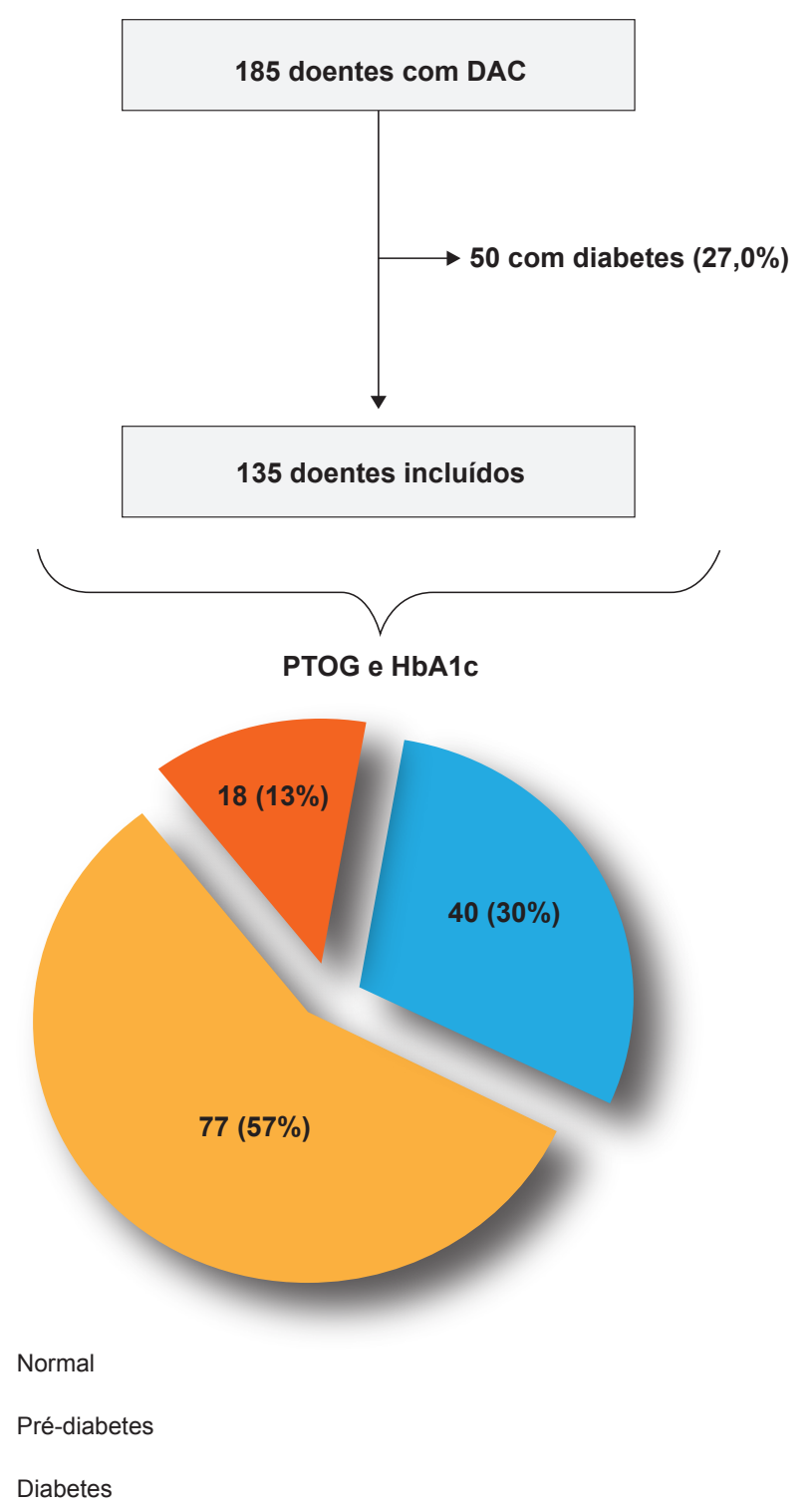

Figura 2 - Flowchart de inclusão de doentes. Prevalência dos distúrbios da glicemia (de acordo com a determinação da HbA1c e da prova de tolerância oral à glucose). 
Tabela 2 - Características da população em função da presença de distúrbios do metabolismo da glicose

\begin{tabular}{|c|c|c|c|c|c|}
\hline & $\begin{array}{l}\text { População Total } \\
(n=135)\end{array}$ & $\begin{array}{l}\text { Glicemia normal } \\
\qquad(n=40)\end{array}$ & $\begin{array}{l}\text { Pré-diabetes } \\
\quad(n=77)\end{array}$ & $\begin{array}{l}\text { Diabetes } \\
(n=18)\end{array}$ & Valor $p$ \\
\hline Idade & $62,3 \pm 13,1$ & $61,3 \pm 13,0$ & $63,0 \pm 13,4$ & $61,4 \pm 12,1$ & $0,755^{*}$ \\
\hline Sexo masculino & $99(73,3)$ & $30(75,0)$ & $55(71,4)$ & $14(77,8)$ & $0,826^{* *}$ \\
\hline \multicolumn{6}{|c|}{ Factores de risco cardiovascular } \\
\hline Índice de massa corporal & $27,6 \pm 4,1$ & $26,5 \pm 3,8$ & $27,8 \pm 4,2$ & $29,3 \pm 2,8$ & $0,041^{*}$ \\
\hline Perímetro abdominal & $98,7 \pm 11,9$ & $96,6 \pm 12,1$ & $98,4 \pm 11,0$ & $104,2 \pm 13,8$ & $0,076^{*}$ \\
\hline Hipertensão arterial & $91(67,4)$ & $28(70,0)$ & $48(62,3)$ & $15(83,3)$ & $0,212^{* *}$ \\
\hline Dislipidemia & $67(49,6)$ & $21(52,5)$ & $34(44,2)$ & $12(66,7)$ & $0,208^{* *}$ \\
\hline Tabagismo & $54(40,0)$ & $22(55,0)$ & $27(35,1)$ & $5(27,8)$ & $0,059^{* *}$ \\
\hline \multicolumn{6}{|l|}{ História prévia } \\
\hline EAM prévio & $21(15,6)$ & $7(17,5)$ & $8(10,4)$ & $6(33,3)$ & $0,050^{* *}$ \\
\hline ICP prévia & $22(16,3)$ & $6(15,0)$ & $10(13,0)$ & $6(33,3)$ & $0,106^{* *}$ \\
\hline \multicolumn{6}{|l|}{ Doença coronária } \\
\hline Fracção de ejecção < 40\% & $13(9,7 \%)$ & $2(5,0)$ & $10(13,2)$ & $1(5,6)$ & $0,301^{* *}$ \\
\hline Doença multivaso & $82(60,7)$ & $21(52,5)$ & $49(63,6)$ & $12(66,7)$ & $0,433^{* *}$ \\
\hline \multicolumn{6}{|l|}{ Perfil lipídico (mg/dL) } \\
\hline Colesterol total & $160 \pm 82$ & $153 \pm 34$ & $164 \pm 45$ & $162 \pm 42$ & $0,406^{*}$ \\
\hline Colesterol HDL & $46 \pm 11$ & $49 \pm 11$ & $45 \pm 11$ & $43 \pm 9$ & $0,148^{*}$ \\
\hline Colesterol LDL & $90 \pm 36$ & $86 \pm 29$ & $92 \pm 40$ & $92 \pm 32$ & $0,658^{*}$ \\
\hline Trigliceridos & $124 \pm 65$ & $96 \pm 36$ & $136 \pm 75$ & $133 \pm 51$ & $0,004^{*}$ \\
\hline \multicolumn{6}{|l|}{ Glicemia e HbA1c } \\
\hline Glicemia em jejum (mg/dL) & $99 \pm 17$ & $88 \pm 6$ & $100 \pm 13$ & $120 \pm 26$ & $<0,001^{*}$ \\
\hline Glicemia 120 min (mg/dL)) & $135 \pm 50$ & $94 \pm 24$ & $139 \pm 34$ & $222 \pm 39$ & $<0,001^{*}$ \\
\hline $\mathrm{HbA1c}(\%)$ & $5,7 \pm 0,5$ & $5,3 \pm 0,2$ & $5,7 \pm 0,3$ & $6,4 \pm 0,015$ & $<0,001^{*}$ \\
\hline
\end{tabular}

Números expressos como média \pm desvio padrão ou $\mathrm{n}(\%) ;{ }^{*}$ Oneway ANOVA; ${ }^{* *} \chi^{2}$

\section{Resultados em função do score de risco de FINDRISC}

$\mathrm{O}$ risco de desenvolver diabetes de acordo com o score FINDRISC foi baixo em 17 (13\%) doentes, ligeiramente elevado em 51 (38\%), moderado em 38 (28\%) e alto/muito alto em 29 (21\%). As características da população em função do score de risco de FINDRISC são apresentadas na Tabela 3. Para além dos parâmetros que estão incluídos no próprio score (índice de massa corporal, perímetro abdominal e antecedentes de hipertensão arterial), não se identificaram outras diferenças significativas entre as populações, nomeadamente no que respeita a outros fatores de risco, aos antecedentes cardiovasculares, às características da doença coronária ou ao perfil lipídico. Também os valores de glicemia em jejum não mostraram diferenças significativas entre os grupos, ao contrário da glicémia aos 120 minutos (PTOG) e a HbA1c, cujos valores foram sucessiva e significativamente subindo de acordo com a subida do score de risco FINDRISC.

\section{Estratégias de diagnóstico dos distúrbios da glicemia (diabetes e pré-diabetes)}

Os resultados das diferentes estratégias de diagnóstico da DM nos doentes com DAC estabelecida são apresentados na Fig. 3A. A estratégia proposta pelas atuais guidelines europeias mostrou uma sensibilidade de $66,7 \%$ e um valor preditivo negativo (VPN) de 95,1\% para o diagnóstico dos novos casos de DM (Kappa 0,78; $p<0,0001$, na comparação com a estratégia PTOG + HbA1c). Utilizando esta estratégia, ficariam por diagnosticar $33 \%$ dos novos casos de DM.

A estratégia de realizar PTOG apenas nos doentes com risco de DM elevado de acordo com o score de FINDRISC, por outro lado, aumentou a sensibilidade para $83,3 \%$ e o VPN para 97,5\% (Kappa 0,85; valor de $p<0,0001$ ). Ainda assim, esta estratégia deixaria por diagnosticar $17 \%$ dos novos casos de DM, em comparação com a estratégia de PTOG + HbA1 em todos os doentes.

No que respeita a todos os casos de distúrbios da glicemia (pré-diabetes e DM), a estratégia proposta pelas guidelines mostrou uma sensibilidade de $87,4 \%$, VPN 76,9\%, enquanto a realização adicional de PTOG nos doentes com FINDRISC elevado melhorou a sensibilidade para $90,5 \%$ e o VPN para $81,6 \%$, ficando ainda $10 \%$ de casos de distúrbios da glicemia por diagnosticar, em comparação com a estratégia PTOG+HbA1c em todos os doentes.

\section{DISCUSSÃO}

Em resumo, os principais resultados deste trabalho foram: 1) a prevalência dos distúrbios do metabolismo da glicemia e, em particular, de diabetes, é muito elevada nos 
Tabela 3 - Características da população em função do score de risco FINDRISC

\begin{tabular}{|c|c|c|c|c|c|}
\hline & $\begin{array}{l}\text { Baixo } \\
(n=17)\end{array}$ & $\begin{array}{l}\text { Lig. elevado } \\
\qquad(\mathrm{n}=51)\end{array}$ & $\begin{array}{l}\text { Moderado } \\
(n=38)\end{array}$ & $\begin{array}{c}\text { Alto } \\
(n=29)\end{array}$ & Valor $p$ \\
\hline Idade $^{\dagger}$ & $59,8 \pm 14,2$ & $61,3 \pm 13,0$ & $62,2 \pm 13,2$ & $65,8 \pm 12,2$ & $0,390^{*}$ \\
\hline Sexo masculino & $16(94,1)$ & $37(72,5)$ & $28(73,7)$ & $18(62,1)$ & $0,130^{\star *}$ \\
\hline \multicolumn{6}{|c|}{ Factores de risco cardiovascular } \\
\hline Índice de massa corporal ${ }^{\dagger}$ & $23,8 \pm 1,8$ & $26,7 \pm 3,7$ & $28,3 \pm 4,1$ & $30,5 \pm 3,5$ & $<0,001^{* *}$ \\
\hline Perímetro abdominal ${ }^{+}$ & $86,2 \pm 7,6$ & $96,9 \pm 12,4$ & $102,2 \pm 10,0$ & $104,4 \pm 9,3$ & $<0,001^{* *}$ \\
\hline Hipertensão arterial ${ }^{\dagger}$ & $4(23,5)$ & $29(56,9)$ & $31(81,6)$ & $27(93,1)$ & $<0,001^{* *}$ \\
\hline Dislipidemia & $6(35,3)$ & $21(41,2)$ & $22(57,9)$ & $22(62,1)$ & $0,128^{* *}$ \\
\hline Tabagismo & $7(41,2)$ & $21(41,2)$ & $19(50,0)$ & $7(24,1)$ & $0,198^{* *}$ \\
\hline \multicolumn{6}{|l|}{ História prévia } \\
\hline EAM prévio & $1(5,9)$ & $6(11,8)$ & $8(21,1)$ & $6(20,7)$ & $0,358^{* *}$ \\
\hline ICP prévia & $1(5,9)$ & $8(15,7)$ & $6(15,8)$ & $7(24,1)$ & $0,444^{* *}$ \\
\hline \multicolumn{6}{|l|}{ Doença coronária } \\
\hline Fracção de ejecção < 40\% & $1(5,9)$ & $3(6,0)$ & $7(18,4)$ & $2(6,9)$ & $0,202^{* *}$ \\
\hline Doença multivaso & $9(52,9)$ & $29(56,9)$ & $23(60,5)$ & $21(72,4)$ & $0,491^{* *}$ \\
\hline \multicolumn{6}{|l|}{ Perfil lipídico (mg/dL) } \\
\hline Colesterol total & $155 \pm 45$ & $163 \pm 38$ & $170 \pm 51$ & $146 \pm 28$ & $0,124^{*}$ \\
\hline Colesterol HDL & $49 \pm 10$ & $46 \pm 11$ & $45 \pm 11$ & $44 \pm 12$ & $0,500^{*}$ \\
\hline Trigliceridos & $93 \pm 35$ & $132 \pm 77$ & $137 \pm 65$ & $111 \pm 47$ & $0,063^{*}$ \\
\hline \multicolumn{6}{|l|}{ Glicemia e HbA1c } \\
\hline Glicemia em jejum (mg/dL) & $97 \pm 11$ & $96 \pm 14$ & $101 \pm 21$ & $103 \pm 18$ & $0,21^{*}$ \\
\hline Glicemia $120 \mathrm{~min}(\mathrm{mg} / \mathrm{dL}))$ & $116 \pm 36$ & $124 \pm 42$ & $144 \pm 50$ & $155 \pm 60$ & $0,01^{*}$ \\
\hline $\mathrm{HbA1c}(\%)$ & $5,5 \pm 0,4$ & $5,6 \pm 0,4$ & $5,8 \pm 0,5$ & $5,9 \pm 0,6$ & $0,007^{*}$ \\
\hline
\end{tabular}

Números expressos como média \pm desvio padrão ou n(\%); ${ }^{\dagger}$ Integra o score de risco; * Oneway ANOVA; ** $\chi^{2}$

Novos casos de diabetes

\begin{tabular}{|c|c|c|c|c|c|}
\hline & Sens & VPN & $\begin{array}{l}\text { \% casos não } \\
\text { diagnosticados }\end{array}$ \\
\hline \multirow{3}{*}{ 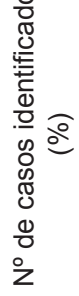 } & 12 & HbA1c + GPJ & 66,7 & $95,1 \%$ & $33 \%$ \\
\hline & 15 & HbA1c + GPJ + PTOG (se FINDRISC elevado) & 83,3 & $97,5 \%$ & $17 \%$ \\
\hline & 18 & PTOG + HbA1c & & & \\
\hline \multicolumn{6}{|c|}{ B Novos casos de diabetes e pré-diabetes } \\
\hline \multirow{3}{*}{ 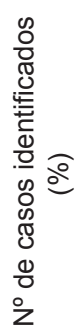 } & 83 & HbA1c + GPJ & 87,4 & $76,9 \%$ & $13 \%$ \\
\hline & 86 & HbA1c + GPJ + PTOG (se FINDRISC elevado) & $90,5 \%$ & $81,6 \%$ & $10 \%$ \\
\hline & 95 & PTOG + HbA1c & & & \\
\hline
\end{tabular}

Figura 3 - Capacidade diagnóstica das várias estratégias na identificação (A) dos novos casos de diabetes; e (B) dos novos casos de distúrbios da glicemia (diabetes e pré-diabetes) 
doentes com DAC; 2) A estratégia diagnóstica proposta pelas novas guidelines europeias identifica apenas uma parte destes novos casos de diabetes e pré-diabetes; 3) A inclusão do score de FINDRISC na seleção dos doentes candidatos a PTOG melhora a capacidade diagnóstica da estratégia proposta pelas guidelines, mas continua a não permitir identificar um número significativo (um em cada seis) de doentes diabéticos.

\section{Prevalência da diabetes/pré-diabetes em doentes com DAC}

A elevada prevalência dos distúrbios do metabolismo da glicemia foi demonstrada de forma inequívoca no Euro Heart Survey on Diabetes. ${ }^{4}$ Neste estudo multicêntrico internacional, era recomendada a realização de uma PTOG em todos os doentes com DAC (estável ou aguda) sem diabetes previamente conhecida. Entre os doentes estáveis que realizaram esta prova, observou-se uma incidência de diabetes de novo ou de pré-diabetes em, respetivamente, $14 \%$ e $37 \%$ dos doentes. Entre os doentes 'agudos', as incidências foram respetivamente de $22 \%$ e $36 \%$. Estes números somaram-se aos $31 \%$ que já tinham diabetes diagnosticada no momento da inclusão. Os autores concluíram que a maioria dos doentes com DAC tem um metabolismo anormal da glucose, recomendado a realização de PTOG em todos os doentes sem diabetes previamente conhecida. Também no nosso estudo confirmámos estes resultados: apenas 40 dos 185 doentes $(21,6 \%)$ inicialmente avaliados apresentava um metabolismo normal da glicemia, sendo que 68 (36,8\%) eram diabéticos (incluindo 50 doentes com diagnóstico de diabetes estabelecido e 18 que desconheciam este diagnóstico) e 77 (41,6\%) pré-diabéticos. Vale a pena salientar que, tal como descrito no Euro Heart Survey on Diabetes, ${ }^{4}$ a prevalência de diabetes que observámos é muito superior à descrita na população Portuguesa geral (13,1\% nos últimos dados conhecidos). ${ }^{17}$ Estes resultados confirmam a absoluta necessidade de rastrear os distúrbios da glucose em todos os doentes com DAC, tendo em atenção o impacto prognóstico que este distúrbio metabólico tem nesta população. Embora não existam dados publicados que permitam avaliar a percentagem de doentes com DAC em que este rastreio é efetuado, a perceção é que serão muito poucos e que a maioria dos médicos (incluindo cardiologistas) que acompanha estes doentes não está sensibilizada para este problema.

\section{Estratégia diagnóstica da diabetes nos doentes com DAC}

As recomendações europeias mais recentes $(2013)^{3}$ introduziram uma alteração muito relevante em relação às recomendações prévias no que diz respeito à estratégia de diagnóstico dos distúrbios da glicemia em doentes com DAC: o diagnóstico passou a ser efetuado apenas com base na GPJ e na HbA1c, sendo a PTOG reservada para os casos 'duvidosos'. Esta foi uma mudança de algum modo surpreendente, se tivermos em consideração que as anteriores recomendações se basearam no já refe- rido Euro Heart Survey on Diabetes, em que a PTOG foi o elemento diagnóstico essencial. ${ }^{10} \mathrm{~A}$ introdução da $\mathrm{HbA} 1 \mathrm{c}$ como critério diagnóstico parece natural, tendo em atenção que este passou a ser um critério diagnóstico na população em geral, ${ }^{16,18,19}$ mas a limitação da PTOG para os casos 'duvidosos' não é claramente fundamentada nas novas guidelines. Aliás, a própria definição de caso 'duvidoso' não é clara nas recomendações, podendo ser interpretada como os doentes com valores laboratoriais equívocos, ou eventualmente os doentes com maior risco de diabetes. Para a avaliação do risco de diabetes, as guidelines sugerem o score de FINDRISC, embora notando que este é um score desenvolvido em populações sem DAC conhecida.

No nosso estudo, a estratégia proposta pelas guidelines permitiu apenas identificar $66 \%$ dos novos casos de diabetes, quando comparada com a realização sistemática de PTOG + HbA1c a todos os doentes. Mesmo selecionando os doentes 'duvidosos' - definidos como aqueles que apresentavam um risco de diabetes (score de FINDRISC elevado ou muito elevado) - para realização de PTOG, apenas $83,3 \%$ dos doentes diabéticos foram corretamente identificados, o que significa que 1 em cada 6 doentes não foi diagnosticado.

Não seria, naturalmente, de esperar que estratégias diagnósticas diferentes identificassem exatamente os mesmos doentes com diabetes. Todas as técnicas têm vantagens e desvantagens e enquanto a PTOG obriga à carga de glucose e a uma segunda colheita de sangue, não sendo absolutamente reprodutível, a HbA1c, embora permita avaliar com maior fiabilidade a exposição crónica à glicose, é um teste mais caro e pode variar com algumas patologias. $^{19}$

Num trabalho prévio, quando as recomendações europeias sugeriam apenas a realização de PTOG, demonstrámos que a HbA1c acrescentava valor diagnóstico, permitindo identificar cerca de $15 \%$ de novos casos não diagnosticados pela PTOG. ${ }^{14}$ Neste trabalho, confirmamos que o contrário também se verifica: a realização de PTOG permite identificar cerca de $17 \%$ de casos de diabetes não identificados pela $\mathrm{HbA} 1 \mathrm{c}$ e pela GPJ. Adicionalmente, a realização da PTOG permite identificar mais $13 \%$ de doentes com distúrbios da glicemia (diabetes e pré-diabetes), quando comparada com a estratégia proposta pelas guidelines (HbA1c + GPJ).

A discussão em torno da realização da PTOG nos doentes com DAC, no entanto, não se resume à sua capacidade de identificar diferentes populações de doentes com diabetes, prendendo-se também com a sua capacidade de identificar os doentes com resistência aumentada à insulina e, em consequência, maior risco de eventos cardiovasculares. ${ }^{20}$

Os critérios diagnósticos atuais, definidos para a população em geral, basearam-se nos níveis de glicemia e $\mathrm{HbA} 1 \mathrm{c}$ que se associam a um aumento do risco de doença microvascular. ${ }^{16} \mathrm{~A}$ doença macrovascular e as suas consequências não foram devidamente tidas em consideração na definição dos critérios de diabetes, tanto mais que 
ensaios recentes (ACCORD, ${ }^{21}$ ADVANCE $^{22}$ ) falharam em demonstrar redução dos eventos macrovasculares com a diminuição intensiva dos valores de glicemia. No entanto, estes resultados devem ser analisados tendo em atenção que a doença microvascular parece associar-se fundamentalmente à diminuição da função das células $\beta$ pancreáticas, enquanto a doença macrovascular (como a doença coronária) parece relacionar-se sobretudo com a diminuição da sensibilidade à insulina. ${ }^{23}$ Sabendo que os estudos ACCORD e ADVANCE avaliaram os eventos em função dos valores de $\mathrm{HbA1c}$ (que representam principalmente a função das células $\beta$ pancreáticas), seria de esperar uma relação mais próxima com a doença microvascular e não surpreende que não se tenha demonstrado uma redução dos eventos macrovasculares.

A sensibilidade à insulina, por outro lado, é melhor avaliada pela realização da PTOG: o estado pós-prandial requer uma rápida e elevada secreção de insulina, que está diminuída nos doentes com resistência à insulina. Nos doentes com DAC, este fenómeno, que não é avaliável pela GPJ ou pelos valores de $\mathrm{HbA} 1 \mathrm{c}$, associa-se a um risco acrescido do risco e de eventos macrovasculares..$^{20,24}$ Adicionalmente, a análise apenas da $\mathrm{HbA} 1 \mathrm{c}$ não permite avaliar outro aspeto importante do metabolismo da glicemia - as oscilações que ocorrem no período pós-prandial nos doentes com resistência à insulina. A oscilação da glicemia tem demonstrado atuar de forma deletéria a vários níveis: ativa a via da proteína cinase $\mathrm{C}$, induz a produção de vários marcadores inflamatórios e determina uma deterioração mais marcada da função endotelial, quando comparada com níveis elevados mas estáveis da glicemia. ${ }^{25-27}$

Em doentes com predomínio de doença macrovascular, a $\mathrm{HbA1c}$ pode, portanto, subestimar o risco associado aos distúrbios da glicemia, fundamentando a utilização rotineira da PTOG nesta população. Tendo em atenção a elevada prevalência e o impacto prognóstico dos distúrbios da glicemia nos doentes com DAC, ${ }^{6,7}$ é no mínimo discutível que se negligencie a informação fornecida por esta técnica, mesmo tendo em consideração o incómodo da carga de glucose e a necessidade de uma segunda colheita de sangue. A identificação precoce da DM e dos estádios de pré-DM é particularmente importante nestes doentes, permitindo a introdução de intervenções farmacológicas ou no estilo de vida ${ }^{28,29}$ que previnam ou atrasem a evolução para diabetes e reduzam o risco das suas complicações macrovasculares. Por fim, é importante realçar que a realização sistemática da PTOG, como defendemos neste trabalho, acarreta um custo adicional muito reduzido: de acordo com a tabela atual de preços laboratoriais do Ministério da Saúde,$^{30}$ a avaliação da glicemia custa $€ 1,20$ (em comparação

\section{REFERÊNCIAS}

1. Saudek CD, Herman WH, Sacks DB, Bergenstal RM, Edelman D, Davidson MB. A new look at screening and diagnosing diabetes mellitus. J Clin Endocrinol Metab. 2008;93:2447-53.

2. Harris MI. Undiagnosed NIDDM: clinical and public health issues. Diabetes Care. 1993;16:642-52

3. Ryden L, Grant PJ, Anker SD, Berne C, Cosentino F, Danchin N, et com $€ 7,30$, para a $\mathrm{HbA} 1 \mathrm{c}$ ) e seria esse o custo adicional por doente em comparação com a estratégia diagnóstica defendida pelas atuais guidelines (glicemia em jejum + $\mathrm{HbA1c})$.

\section{Limitações}

Este é um trabalho de um único centro e com uma população selecionada de pequena dimensão, com doença coronária clinicamente expressiva - com indicação para angioplastia, pelo que os resultados devem ser interpretados nesse contexto. Adicionalmente, os testes de diagnóstico da diabetes não foram repetidos, como recomendado pelas guidelines, as quais sugerem que o diagnóstico não deve basear-se apenas numa medição da glicemia ou da HbA1c.

\section{CONCLUSÃO}

A estratégia proposta nas novas guidelines identifica apenas $66,7 \%$ dos novos casos de DM, deixando por diagnosticar uma importante percentagem de doentes. Utilizar o score de risco de FINDRISC como forma de selecionar os doentes candidatos a rastreio com PTOG, melhora a capacidade diagnóstica quando comparada com a simples utilização da GPJ em conjunto com a HbA1c. Porém, esta estratégia deixa por diagnosticar $17 \%$ destes doentes, o que significa que 1 em cada 6 doentes não é diagnosticado. Tendo em atenção o impacto prognóstico da DM na DAC, a identificação de todos os casos é fundamental, pelo que deveria, tendo em conta os resultados deste estudo, continuar a recomendar-se a realização sistemática de PTOG em todos os doentes com DAC e sem DM previamente conhecida.

\section{PROTECÇÃO DE PESSOAS E ANIMAIS}

Os autores declaram que os procedimentos seguidos estavam de acordo com os regulamentos estabelecidos pelos responsáveis da Comissão de Investigação Clínica e Ética e de acordo com a Declaração de Helsínquia da Associação Médica Mundial.

\section{CONFIDENCIALIDADE DOS DADOS}

Os autores declaram ter seguido os protocolos do seu centro de trabalho acerca da publicação de dados.

\section{CONFLITOS DE INTERESSE}

Os autores declaram não terem qualquer conflito de interesse relativamente ao presente artigo.

\section{FONTES DE FINANCIAMENTO}

Os autores declaram não ter recebido subsídios ou bolsas para a elaboração do artigo

al. ESC Guidelines on diabetes, pre-diabetes, and cardiovascular diseases developed in collaboration with the EASD: the Task Force on diabetes, pre-diabetes, and cardiovascular diseases of the European Society of Cardiology (ESC) and developed in collaboratio. Eur Heart J. 2013;34:3035-87.

4. Bartnik M, Ryden L, Ferrari R, Malmberg K, Pyörälä K, Simoons M, et al 
The prevalence of abnormal glucose regulation in patients with coronary artery disease across Europe. The Euro Heart Survey on diabetes and the heart. Eur Heart J. 2004;25:1880-90.

5. Wang JS, Lee IT, Lee WJ, Lin SY, Fu CP, Ting CT, et al. Performance of $\mathrm{HbA} 1 \mathrm{c}$ and fasting plasma glucose in screening for diabetes in patients undergoing coronary angiography. Diabetes Care. 2013;36:1138-40.

6. Bartnik M, Malmberg K, Norhammar A, Tenerz A, Ohrvik J, Ryden L. Newly detected abnormal glucose tolerance: an important predictor of long-term outcome after myocardial infarction. Eur Heart J. 2004;25:1990-7.

7. Doerr R, Hoffmann U, Otter W, Heinemann L, Hunger-Battefeld W, Kulzer $\mathrm{B}$, et al. Oral glucose tolerance test and $\mathrm{HbA}(1) \mathrm{c}$ for diagnosis of diabetes in patients undergoing coronary angiography: [corrected] the Silent Diabetes Study. Diabetologia. 2011;54:2923-30.

8. de Vegt F, Dekker JM, Ruhe HG, Stehouwer CD, Nijpels G, Bouter LM, et al. Hyperglycaemia is associated with all-cause and cardiovascular mortality in the Hoorn population: the Hoorn Study. Diabetologia. 1999;42:926-31.

9. Glucose tolerance and mortality: comparison of WHO and American Diabetes Association diagnostic criteria. The DECODE study group. European Diabetes Epidemiology Group. Diabetes Epidemiology: Collaborative analysis Of Diagnostic criteria in Europe. Lancet. 1999;354:617-21.

10. Ryden L, Standl E, Bartnik M, Van den Berghe G, Betteridge J, de Boer MJ, et al. Guidelines on diabetes, pre-diabetes, and cardiovascular diseases: executive summary. The Task Force on Diabetes and Cardiovascular Diseases of the European Society of Cardiology (ESC) and of the European Association for the Study of Diabetes (EASD). Eur Heart J. 2007;28:88-136

11. Sacks DB. A1C versus glucose testing: a comparison. Diabetes Care. 2011;34:518-23.

12. Sattar N, Preiss D. HbA1c in type 2 diabetes diagnostic criteria: addressing the right questions to move the field forwards. Diabetologia. 2012;55:1564-7.

13. de Mulder M, Oemrawsingh RM, Stam F, Boersma E, Umans VA. Comparison of diagnostic criteria to detect undiagnosed diabetes in hyperglycaemic patients with acute coronary syndrome. Heart. 2012;98:37-41

14. Baptista S, Abreu P, Gil V. Impact of glycosylated hemoglobin evaluation on the diagnosis of glucose metabolism abnormalities in patients with coronary artery disease. J Am Coll Cardiol. 2012;59:E1665.

15. Lindstrom J, Tuomilehto J. The Diabetes Risk Score: a practical tool to predict type 2 diabetes risk. Diabetes Care. 2003;26:725-31.

16. Classification and diagnosis of diabetes. Diabetes Care. 2016;39:S1112

17. Relatório Anual do Observatório Nacional da Diabetes - "Diabetes: Factos e Números", Edição de 2015. [consultado 2016 jun 16]. Disponível em: https://www.dgs.pt/estatisticas-de-saude/estatisticasde-saude/publicacoes/diabetes-factos-e-numeros-7-edicao-pdf.aspx.
2015;

18. World Health Organization. Use of glycated haemoglobin $(\mathrm{HbA} 1 \mathrm{c})$ in the diagnosis of diabetes mellitus. [consultado 2016 jun 16]. Disponível em: http://www.who.int/diabetes/publications/diagnosis_diabetes2011/en/ index.html

19. Gillett MJ. International Expert Committee report on the role of the A1c assay in the diagnosis of diabetes: Diabetes Care. 2009; 32: 1327-34. Clin Biochem Rev. 2009;30:197-200.

20. Sourij H, Saely CH, Schmid F, Zweiker R, Marte T, Wascher TC, et al. Post-challenge hyperglycaemia is strongly associated with future macrovascular events and total mortality in angiographied coronary patients. Eur Heart J. 2010;31:1583-90.

21. ACCORD. Effects of intensive glucose lowering in type 2 diabetes. $N$ Engl J Med. 2008;358:2545-59.

22. ADVANCE. Intensive blood glucose control and vascular outcomes in patients with type 2 diabetes. N Engl J Med. 2008;358:2560-72.

23. Mulvey CK, McNeill AM, Girman CJ, Churchill TW, Terembula K, Ferguson JF, et al. Differential associations of oral glucose tolerance test-derived measures of insulin sensitivity and pancreatic beta-cell function with coronary artery calcification and microalbuminuria in type 2 diabetes. Diabetes Care. 2014;37:124-33.

24. Schutt M, Schmidt T, Schwaab B. Cardio-metabolic characterisation of patients with coronary heart disease: specific consideration of the 1-h postprandial plasma glucose. Exp Clin Endocrinol Diabetes. 2012;120:435-8.

25. Quagliaro L, Piconi L, Assaloni R, Martinelli L, Motz E, Ceriello A. Intermittent high glucose enhances apoptosis related to oxidative stress in human umbilical vein endothelial cells: the role of protein kinase $\mathrm{C}$ and $\mathrm{NAD}(\mathrm{P}) \mathrm{H}$-oxidase activation. Diabetes. 2003;52:2795-804.

26. Azuma K, Kawamori R, Toyofuku Y, Kitahara Y, Sato F, Shimizu T, et al. Repetitive fluctuations in blood glucose enhance monocyte adhesion to the endothelium of rat thoracic aorta. Arterioscler Thromb Vasc Biol. 2006;26:2275-80.

27. Quagliaro L, Piconi L, Assaloni R, Da Ros R, Maier A, Zuodar G, et al. Intermittent high glucose enhances ICAM-1, VCAM-1 and E-selectin expression in human umbilical vein endothelial cells in culture: the distinct role of protein kinase $\mathrm{C}$ and mitochondrial superoxide production. Atherosclerosis. 2005;183:259-67.

28. Tuomilehto J, Lindstrom J, Eriksson JG, Valle TT, Hämäläinen H, llanneParikka $P$, et al. Prevention of type 2 diabetes mellitus by changes in lifestyle among subjects with impaired glucose tolerance. N Engl J Med. 2001;344:1343-50.

29. Knowler WC, Barrett-Connor E, Fowler SE, Hamman RF, Lachin JM Walker EA, et al. Reduction in the incidence of type 2 diabetes with lifestyle intervention or metformin. N Engl J Med. 2002;346:393-403.

30. Tabela de meios complementares de diagnóstico e terapêutica convencionados. [consultado 2016 jun 16]. Disponível em: http://www. acss.min-saude.pt/2016/10/03/tabela-mcdt/. 2016; 